\title{
User related factors in implementation of WHO surgical safety checklist (SSC)
}

\author{
Amadoru SDS, ${ }^{1}$ Perera KYD, ${ }^{1}$ Amjed DMMLA, ${ }^{1}$ Chaminda JLP, ${ }^{1}$ Kandamby HGIT ${ }^{2}$ \\ 1. Registrar (Medical Administration), Post Graduate Institute of Medicine, Colombo \\ 2. Medical officer, District General Hospital, Matara
}

Corresponding Author: S D S Amadoru

sanjeewaamadoru@gmail.com

https://orcid.org/0000-0001-5491-560X

\begin{abstract}
Introduction: WHO Surgical Safety Checklist (SSC) was introduced to address the well-recognized global problem of lack of surgical safety. Although proper implementation of SSC reduces common adverse events occurring during surgical procedures, the practical implementation in some cases has been found to be less than optimal.
\end{abstract}

Objective: To assess the user related factors in the implementation of the SSC at District General Hospital (DGH), Matara

Methods: A descriptive cross-sectional study was carried out at DGH, Matara using a self-administered questionnaire among 76 doctors and 116 nurses who worked in operating theatres of DGH,Matara. A questionnaire based on the SSC and its implementation was used to assess the knowledge, attitudes and practices of doctors and nurses.

Results: On the average,the seniority and experience in working in the operating theatres of DGH,Matara had a significant association with the awareness of the SSC. Nurses were more familiar about the SSC compared to doctors. Even when the user of the checklist had good knowledge, favorable attitudes and interest for proper implementation of the SSC, only a minority (8.7\%) was trained in SSC.

Discussion: The complexity, uncertainty of the definition of the responsibility and frequent unavailability of checklists were the main perceived barriers. It is recommended to simplify and reformat the checklist to make more user friendly and designating a checklist coordinator, training and monitoring implementation for better compliance.

(Key words: Patient safety, Surgical Safety Checklist, adverse events)

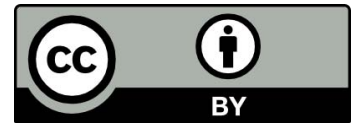

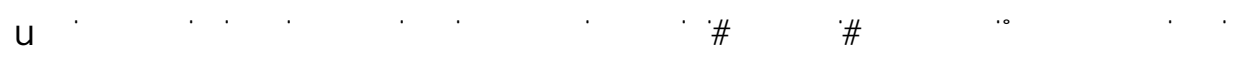

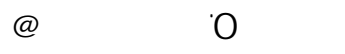




\section{Introduction}

Patient safety has received much attention globally in recent years due to increasing incidence of adverse events during healthcare delivery. Surgical safety can be considered as an integral component of patient safety. Because of high incidence of injuries due to trauma, malignancies and cardiovascular disease, the volume of surgery is growing. According to World Health Organization statistics, a number of 234 million major surgeries are done each year around the world. This corresponds to one out of 25 people undergoing surgical intervention every year. ${ }^{1}$

Performing a surgery itself carries a risk. Although saving lives is the intention of surgery, unsafe surgery can cause severe harm. ${ }^{2}$ Every year 7 million people around the world suffer complications due to surgery. Approximately one million die due to surgical complications which is almost twice the incidence of maternal deaths. It is considered that half of these cases are potentially preventable. ${ }^{3}$ Lack of surgical safety is a well-recognized global problem. Therefore, a global initiative to promote safer surgery could save millions of lives worldwide. ${ }^{4}$

WHO working group on patient safety with the objective to guide the operating teams in reducing the number of surgical complications developed the surgical safety checklist. ${ }^{5}$ The aim of the surgical safety checklist is to ensure incorporation of key safety elements to the operating theatre with minimum burden to the staff and the system.

With the implementation of the checklist, the operating team will follow safety steps and minimize the commonest risks which are avoidable which endanger lives of patients. ${ }^{6,12}$

According to available literature, many surgical mishaps could have been saved by a well-maintained surgical safety checklist. A multicenter pilot study carried out after the introduction of WHO surgical safety checklist in eight hospitals in different parts of the world revealed a marked improvement in outcomes of surgical procedures. The death rate and post-operative complications fallen by $36 \%{ }^{7}$

Although there are known benefits of the surgical safety checklist, the practical utilization in some cases has been found to be less than expected. 1,022,941 surgeries had been performed island wide in Sri Lanka including 396,534 major surgeries during the year 2015. In the same year, 32,877 surgeries performed in Matara District including 10,229 major surgeries. ${ }^{8}$ In 2016, there were 120,533 admissions to the District General Hospital, Matara. Total number of operations performed in year 2016 was 30,978 . This illustrates that $25 \%$ of patients admitted to the hospital had undergone surgery. ${ }^{14}$

Litigation of caregivers due to malpractice is increasing. Probably the real situation is even worse because most incidents are not reported. It is evident that proper implementation of a surgical safety checklist could be a major component in ensuring patient safety. ${ }^{9,13}$

On $25^{\text {th }}$ of June 2008 , the WHO surgical safety checklist was officially launched in Washington DC, United States of America, to promote its implementation globally. Ministry of Health Sri Lanka launched the surgical safety checklist with some modifications on $24^{\text {th }}$ December 2013.The Secretary of Health has issued a circular (No: 02-185/2013, 23-12-2013) to implement the surgical safety checklist for all operations in all hospitals island wide. ${ }^{10}$ 
By introducing the checklist, the surgical process is divided into three main phases, which corresponds to the time period in the normal flow of a surgical procedure -

- Sign in - before induction of anesthesia

- Time out - after induction of anesthesia and before surgical incision.

- Sign out - during or immediately after wound closure and before removing the patient from the operating room

Designating a single person for confirmation of checklist completion is necessary, as some steps may be overlooked in a complex setting of an operating room. ${ }^{11}$ The checklist coordinator must be allowed to confirm that in each phase, the surgical team has completed its current task before it proceeds further. ${ }^{5}$ WHO working group on patient safety emphasizes that the institutions have the freedom of modifying the WHO surgical safety checklist according to the culture of the operating theatre, availability of facilities and the degree of familiarity among members of the operating team. ${ }^{5}$

\section{Objective}

To assess the user related factors in implementation of the Surgical Safety Checklist at District General Hospital, Matara.

\section{Methodology}

A hospital based descriptive cross-sectional study was carried outfrom January 2017 to May 2017 using a structured self-administered questionnaire among the whole population of 76 doctors and 116 nurses who worked in the four operating theatres of DGH, Matara. The questionnaire was based on the SSC and its implementation to assess the knowledge, attitudes and practices of doctors and nurses. Fivepoint Likert scale was used wherever relevant.

Ethical clearance was obtained from the Ethics Review Committee of the Post Graduate Institute of Medicine. Absolute confidentiality of the information was assured and the informed consent was obtained from the voluntary participants.

Data were coded and entered into a database and data clearing was done and appropriate descriptive and analytical techniques were used in data analysis. Data analysis was done using SPSS software. Chisquare test was applied wherever relevant. Level of significance was set at 0.05 .

\section{Results}

Sixty-nine (69) doctors and one hundred and five (105) nurses responded to the questionnaire withoverall response rate of $90.6 \%$. The age category with highest representation of both doctors and nurses was 35 to 44 years age group. One third of Doctors who worked in the operating theatres at DGH Matara were anesthetists and one third of the nurses worked in the General Surgery Theatre (OT B). Nearly half the number of doctors and three quarter of nurses had more than 10 years of service.

It was noticed that all the nurses have heard about the surgical safety checklist while only $79 \%$ ( $n=55)$ doctors have heard about it. Further, there was a statistically significant association between the Theatre experience and the awareness of the SSC.Majority of nurses anddoctors had got to know about the checklist by working in the operating theatre $(83.8 \%, n=88$ and $71.0 \%, n=49$ respectively). Majority of doctors and nurses were unaware about the General circular 01-185/2013 issued by the Secretary of Health on implementation of the surgical safety checklist which was as high as $75.5 \%(n=52)$ in doctors and $62.9 \%(n=66)$ in nurses. Almost half of the doctors and nurses were aware that the surgical safety checklist was developed by World Health Organization to be implemented globally. 
Table 1: Familiarity of surgical safety checklist among participants

\begin{tabular}{|c|c|c|c|c|c|}
\hline \multirow{2}{*}{$\begin{array}{l}\text { Familiarity } \text { of } \\
\text { surgical safety } \\
\text { checklist }\end{array}$} & \multicolumn{2}{|l|}{ Doctors } & \multicolumn{2}{|l|}{ Nurses } & \multirow[t]{2}{*}{ Significance } \\
\hline & Frequency & Percentage & Frequency & Percentage & \\
\hline $\begin{array}{l}\text { Completely } \\
\text { unfamiliar }\end{array}$ & 17 & $25.0 \%$ & 15 & $14.6 \%$ & $x^{2}=12.35 d f=2$ \\
\hline Partially familiar & 40 & $58.8 \%$ & 84 & $81.6 \%$ & $P=0.002$ \\
\hline $\begin{array}{l}\text { Completely } \\
\text { familiar }\end{array}$ & 11 & $16.2 \%$ & 4 & $3.8 \%$ & \\
\hline Total & $68 *$ & $100.0 \%$ & $103^{*}$ & $100.0 \%$ & \\
\hline
\end{tabular}

*One doctor and two nurses not responded

Table 1 shows nurseswere morefamiliar about the surgical safety checklist than the doctors. Eighty percent of nurses were partially familiar with the checklist. The difference observed was statistically significant $[p<0.05]$. Only $13 \%$ of doctors and $5 \%$ of nurses received training on surgical safety checklist before it was introduced in the institution.

More than $80 \%$ of Doctors and Nurses knew that the surgical safety checklist would improve the safety of both patients and the staff.All nurses and $95 \%$ of doctors knew that the responsibility in maintaining the surgical safety checklist is with all members of the team including surgeon, anesthetist and nurses.

Majority of doctors were of the opinion that a checklist should be used even in an emergency ( $n=47$, 69.1\%). However, the nurse's opinion was equal (50\%) for maintaining for both routine and emergency operations. 57\%, $(n=61)$ of nurses were in the opinion that the checklist has to be memorized. However, the majority $(80.0 \%, n=52)$ of doctors correctly identified that it need not to be memorized.

Almost two third of the doctors and nurses had experienced a surgical complication or a death which they thought could have been prevented. Majority of doctors and $(89.8 \%, n=61)$ and nurses $(89.4 \%, n=$ 93) were of the opinion that operating on the wrong patient and wrong site can be prevented by proper maintenance of surgical safety checklist.

$50 \%$ of doctors and nurses were of the opinion that it is difficult to maintain a checklist with current workload and causes unnecessary delay to operation list. However, majority (80\%) of them were of the opinion that it is worthwhile to spend few minutes to complete the checklist even in an emergency.

Importantly, three quarter $(75 \%, n=52)$ of doctors and $91 \%(n=95)$ of nurses were of the opinion that they need in-service training in surgical safety checklist.

Table 2: Deficiencies of surgical safety checklist identified by the participants

Checklist related factors

Checklist is too long and too complex

Checklist includes unnecessary components

Unsuitable structure of the checklist

Most of the checks are already practiced 


\section{User related factors}

Difficult to maintain due to overwork

Uncertainty of the responsibility

Poor corporation and conflicts among the staff

Lack of awareness and poor attitudes

\begin{tabular}{l} 
Management related factors \\
No proper introduction of the checklist \\
Lack of training on checklist \\
Frequent unavailability of checklists \\
Lack of infrastructure facilities needed \\
\hline
\end{tabular}

\section{Discussion}

The study revealed that the users had favorable attitudes and interest for proper implementation of the surgical safety checklist. However, they need further training as was highlighted by the participants.

There was a clear gender difference between doctors and nurses, where $91.4 \%$ of nurses were females while $63.8 \%$ of doctors were males. This correlates to the normal population of nurses where more female nurses were produced from the nursing schools. However, there was a clear male dominance of doctors working in the operating theatre.

The statistically significant difference on awareness of the surgical safety checklist and the experience in the operating theatre which indicates that age, theatre experience and teamwork plays an important role in information transfer.Surprisingly, one quarter of the doctors was completely unfamiliar about the surgical safety checklist and only a minority of the doctors and nurses were completely familiar. For proper implementation of the checklist, all surgical team members should be completely familiar with the surgical safety checklist. It was observed that only a very few doctors and nurses $13.0 \%$ and $5.8 \%$ respectively) received training on surgical safety checklist.

Majority of the doctors and nurses appreciated the importance of maintaining a checklist for every operation. However, there was a conflict of ideas between doctors and nurses in maintaining it for minor operations. It was observed that $78.0 \%$ nurses were of the opinion that a checklist need not be used for minor operations, while $53.0 \%$ of doctors were of the opinion that it is needed. Almost all doctors and nurses perceive that the responsibility of maintaining the checklist is with all the team members including the surgeon, anesthetist and the nurse (91.5\% and $100.0 \%$ respectively). More than $75 \%$ of the participants disagreed with the statement that the person maintaining the checklist is solely responsible for the complications which could have been prevented and it should be a team work.

Majority of participants were of the opinion that the surgical safety checklist currently used at DGH Matara was too long and complex and they also worried that too many checks makes the patients fear surgery. 


\section{Conclusion}

On the average, more senior and experienced staff worked in the Operating Theatres at District General Hospital, Matara.The users of the checklist, namely the doctors and nurses who worked in the Operating Theatres of district General Hospital, Matara had a good awareness about the surgical safety checklist. They had favorable attitudes and interest for proper implementation and maintenance of the surgical safety checklist. However, they lacked training in proper maintenance of the checklist as reflected by their practices.

The main barriers in checklist implementation were uncertainty of definition of responsibility in maintaining the checklist, complexity of the checklist and frequent unavailability of the surgical safety checklists.

\section{Recommendations}

It is recommended to simplify the surgical safety checklist guided by the WHO recommendations. Changing the current format of the checklist with separate columns for each phase, aligning the checkboxes and using appropriate fonts and colors are needed to make it appealing and user friendly. A distinct colored paper should be used for easy location of the checklist.

Designating a checklist coordinator who is responsible for maintaining the surgical safety checklist and promoting teamwork for checklist completioncould be a good practice.Establish and maintain a record keeping, monitoring and feedback mechanism on surgical safety checklist and regular discussions on near misses, adverse events and events which could have been prevented by proper maintenance of checklists and establish and maintain a proper communication system for better information flowis essential for proper implementation of the SSC. Continuous training programs should be arranged on maintenance of surgical safety checklist for doctors and nurses working in Operating Theatres.Surgical safety checklist should be assigned a " $\mathrm{H}$ " number and distributed with other printed forms.

\section{References}

1. World Alliance for Patient Safety. WHO Guidelines for Safe Surgery. Safe surgery Saves Lives. World Health Organization. Geneva. 2008.

2. Weiser TG, Regenbogen SE, Thompson KD. A surgical safety checklist to reduce morbidity and mortality in a global population. N Engl J Med. 2009; 360(5): 491-9.

3. Haynes $A B$, Thomas GW, William RB. Changes in safety attitude and relationship to decrease post-operative morbidity and mortality following implementation of a checklist based surgical safety intervention. BMJ Qualsaf. 2011; 20: 102-107.

4. Amarapathy $M$, Sridharan S, Perera R, Handa Y. Factors affecting patient safety culture in a tertiary care hospital in Sri Lanka. International Journal of Scientific and Technology Research. 2013; 2(3):173-180.

5. WHO. Implementation Manual, Surgical Safety Checklist. World Health Organization, Geneva. 2009.

6. Stephanie R. Shantann $r$, Nick $S$, et al. Does safety checklist improve team work and communication in the operating room? Annals of Surgery. 2013; Vol.50(50).

7. Mehrdad A, Farided K, Charles JP. Effects of surgical safety checklist on post-operative morbidity and mortality rates. Quality management and Healthcare. 2011.

8. Ministry of Health Sri Lanka. Health Facility Survey. District Profile. 2015. 
9. Caprice C, Micheal LG, Emillie MR, Thomas et al. A prospective study of patient safety in the operating room. J. surgery. 2005.

10. Ministry of Health Sri Lanka. Implementation of Surgical Safety Checklist. General Circular No; 02-185/2013.

11. Borchard A, Schwappach DLB, Barbir A, Bezzola P. A systematic review of the effectiveness, compliance and critical factors for implementation of safety checklist in Urgery. Ann surg. 2012; 256(6): 925-37.

12. Cavillini GA, Campi I, Mari M,Forlin M. Clinical risk management in eye outpatient surgery.A New surgical safety checklist for cataract surgery and intravitreal anti-VEGF injection.Grates Arch ClinExpOphthalmol. 2012; 251(3): 889-94.

13. Christofer R, Gerd J, Odenrick P, Kristina A, Andres PL. Compliance with the WHO surgical safety checklist: deviations and possible improvements. International Journal for Quality in Health Care. 2013; 25(2): 182-187.

14. Ministry of Health Sri Lanka. Annual Health Bulletin. 2014. 